\title{
Pulse width reduction in single mode diode lasers via external injection of optical pulses
}

\author{
Claudio R. Mirasso ${ }^{\text {a) }}$ \\ Departament de Física, Universitat de les Illes Balears, E-07071 Palma de Mallorca, Spain
}

(Received 16 September 1994; accepted for publication 27 January 1995)

\begin{abstract}
We propose and numerically study a simple scheme to obtain large pulse-width reduction of optical pulses generated by semiconductor lasers. The scheme consists of modulating two single-mode semiconductor lasers coupled through a normal dispersion fiber. The optimal pulse compression is obtained for a particular range of phase delay between the two modulated electrical injection currents. In addition, the optical pulses so obtained have a larger output power and are very close to transform-limited as required for soliton communication systems. (C) 1995 American Institute of Physics.
\end{abstract}

Short pulses produced by high speed semiconductor lasers $^{1-12}$ are essential for a large variety of applications. To date, the strongest demand for picosecond laser diodes remains in the field of optical communications, where transform-limited pulses are usually required. ${ }^{3,4}$ Several mechanisms have been proposed for obtaining short width optical pulses. A simple way to produce them is by gainswitching the semiconductor laser. ${ }^{1-6}$ In this case optical pulses of some picosecond width can be obtained at $\mathrm{GHz}$ rates. However, the pulses so obtained are far from transform-limited, i.e. the product $\tau \Delta \nu \gg 0.315$ (being $\tau$ $(\Delta \nu)$ the FWHM of the optical pulse (spectrum)), since a frequency down-chirp inevitably occurs due to carrier density modulation. Probably the most efficient mechanism to obtain very short optical pulses is the mode-locking technique. ${ }^{1,2,7-9}$ Pulses as short as few femtoseconds can be obtained. However, it is more complex than the gainswitching and has the limitation of a fixed modulation rate. The $Q$-switching technique is also used to obtain short-width pulses $^{1,2,10}$ but it is difficult to provide fast gain modulation.

In this letter we propose a simple scheme to produce short optical pulses by coupling two similar $1.55 \mu \mathrm{m}$ singlemode semiconductor lasers through a normal dispersive fiber. The proposed scheme is shown in Fig. 1. Both laser 1, called the master laser (ML), and laser 2, called slave laser (SL), are considered to have the same parameters and are modulated through a sinusoidal current of the same amplitude, with the only difference that we allow for a temporal phase shift between them $\left(\Delta t_{m s}\right)$. The modulation frequency of the injection current is taken as $5 \mathrm{GHz}$ fixed for both lasers. When the two lasers are decoupled optical pulses of a width of $\sim 37 \mathrm{ps}$ are numerically obtained. However, when the two lasers are coupled and the injection currents are properly phase-shifted, pulses as short as 9.2 ps can be numerically obtained. In addition, these pulses have larger power and are very close to transform-limited as required for soliton transmission systems.

We describe the transient response of the laser in term of the noise driven rate equations for the electric field and carrier number inside the cavity ${ }^{11}$

$$
\begin{aligned}
\frac{d E_{m, s}}{d t}= & (1-j \alpha)\left(\frac{g\left(N_{m, s}-N_{0}\right)}{\left(1+s P_{m, s}\right)}-\frac{1}{\tau_{p}}\right) \frac{E_{m, s}}{2} \\
& +\kappa_{m} E_{m} e^{j t \Delta \omega}+\sqrt{2 \beta N_{m, s}} \xi_{m, s}(t), \\
\frac{d N_{m, s}}{d t}= & \frac{I_{m, s}(t)}{e}-\frac{1}{\tau_{n}} N_{m, s}-\frac{g\left(N_{m, s}-N_{0}\right)}{\left(1+s P_{m, s}\right)} P_{m, s}, \\
I_{m, s}(t)= & A \sin \left(2 \pi \nu t+\varphi_{m, s}\right)+B,
\end{aligned}
$$

where the subindices $m, s$ stands for the ML and SL, respectively. $P_{m, s}=E_{m, s} E_{m, s}^{*}$ is the light intensity, $g=1.5 \times 10^{-8}$ $\mathrm{ps}^{-1}$ is the gain parameter, $s=2 \times 10^{-7}$ is the gain saturation factor, $\alpha=5$ is the linewidth enhancement factor, $\tau_{p}=2 \mathrm{ps}$ is the photon lifetime, $\tau_{n}=2 \mathrm{~ns}$ is the carrier lifetime, $N_{0}=1.485 \times 10^{8}$ is the carrier number at transparency, $\beta=5 \times 10^{-9} \mathrm{ps}^{-1}$ is the spontaneous emission rate, $e=1.602 \times 10^{-19} \mathrm{C}$ is the electronic charge, $\kappa_{m}=0.21 \mathrm{ps}^{-1}$ is the coupling parameter of the injected field and $\Delta \omega=0$ is the detuning parameter. The random spontaneous emission process is modeled by a complex Gaussian white noise term $\xi(t)$ of zero mean and correlation $\left\langle\xi(t) \xi^{*}\left(t^{\prime}\right)\right\rangle$ $=2 \delta\left(t-t^{\prime}\right)$. The sinusoidal injection current of Eq. (3) has a maximum value $A+B=3.5 I_{\text {th }}$, being $I_{\text {th }} \approx 14 \mathrm{~mA}$ the threshold current, and a minimum value $-A+B=0.5 I_{\text {th }}$; $\nu=5 \mathrm{GHz}$ is the modulation frequency of the injection currents; $\varphi_{m}=0$ and $\varphi_{s}=2 \pi \nu \Delta t_{m s}$ is a free parameter running

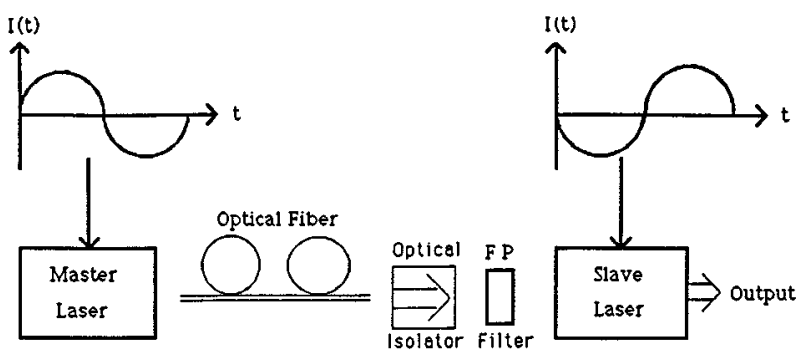

FIG. 1. Proposed scheme for pulse-width reduction. a) Present Address: Instituto de Estructura de la Materia, CSIC, c/ Serrano 123, E-28006, Madrid, Spain. Electronic mail: emclaudio@iem.csic.es 
from $-\pi$ to $\pi$. Equations (1) and (2) are written in the frame of reference where the $\mathrm{cw}$ frequency of the free running laser is zero.

As mentioned above, when the injection current of the ML is modulated at $5 \mathrm{GHz}$ optical pulses of a width of $\sim 37$ ps with a maximum output power (MOP) of $\sim 20 \mathrm{~mW}$ are numerically obtained with these laser parameters from Eqs. (1)-(3) (assuming $\kappa_{m}=0$ ). These pulses are injected in a normal dispersion fiber of $L=3 \mathrm{~km}$ length, dispersion coefficient $\beta_{2}=25 \mathrm{ps}^{2} \mathrm{~km}^{-1}$ and fiber loss of $0.25 \mathrm{~dB} / \mathrm{km}$. This length is the one that produces the maximum compression of these optical pulses. $n_{g}=1.462$ is the group index. With these values of $L$ and $n_{g}$ the time a pulse takes to reach the end of the fiber $t=L n_{g} / c$, where $c=3 \times 10^{5} \mathrm{~km} / \mathrm{sec}$ is the speed of light in vacuum, is exactly an integer number of the modulation period of the injection current $T=200 \mathrm{ps}$. Perfect coupling between the ML and the fiber is assumed and non linear effects in the fiber are neglected. Numerically, the electric field at the output of the fiber is obtained by solving the equation for the pulse propagation in a dispersive regime $^{12}$ and using a fast-Fourier transform algorithm. At the output of the fiber a Fabry-Pérot filter is placed to clean-up the pulses. The bandwidth of the filter is taken as $18 \mathrm{GHz}$ and its central frequency close to the frequency of the free running laser under $\mathrm{cw}$ operation (we used $15 \mathrm{GHz}$ above this frequency in the simulations). An isolator is also placed between the fiber and the filter to prevent feedback towards the ML. Compressed and filtered pulses are then injected into the slave laser.

After compression and filtering the pulses have a width of $\sim 10 \mathrm{ps}$ and a MOP of $\sim 28 \mathrm{~mW}$. These pulses are injected into the SL. The shift parameter of the injection current of the SL $\varphi_{s}$ plays a crucial role in the SL dynamics. We have numerically observed that for a range of $\varphi_{s}$ between $\sim-100^{\circ}$ and $-10^{\circ}$ pulses as short as $\sim 9$ ps width and maximum output power of $\sim 65 \mathrm{~mW}$ can be obtained from the SL. Although the range of $\varphi_{s}$ is large, the better pulses are obtained around $\varphi_{s}=-38^{\circ}$. In Fig. 2 we plot the light pulses at the output of the ML and at the output of the SL for three different $\varphi_{s}$, obtained from numerical integration of Eqs. (1)-(3). As can be clearly seen better pulses are obtained for $\varphi_{s}=-38^{\circ}$. Out of the range of $\varphi_{s}$ between $-100^{\circ}$ and $-10^{\circ}$ the injected pulse passes through the SL with almost no interaction with it and the SL emits its own pulse yielding two pulses in the same period.

The mechanisms that yield this large pulse width reduction can be understood by looking the Fig. 3 (b) where we plot the dynamical behavior of the SL. It is clear that the best delay $\varphi_{s}\left(-38^{\circ}\right)$ is the one in which the time the carriers reach their maximum value is very close to the time the injected pulse reaches its maximum. In this case the carriers recombine more quickly given rise to a strong pulse width reduction. For $\varphi_{s}=-100^{\circ}$ the injected pulses come into the SL at a time at which the carriers have just crossed threshold. Part of them recombine but another part still grows giving rise to a second smaller peak as can be seen in Fig. 2(b). The situation for $\varphi_{s}=-10^{\circ}$ is the contrary, i.e. the injected pulses come into the SL when the carriers are reaching their
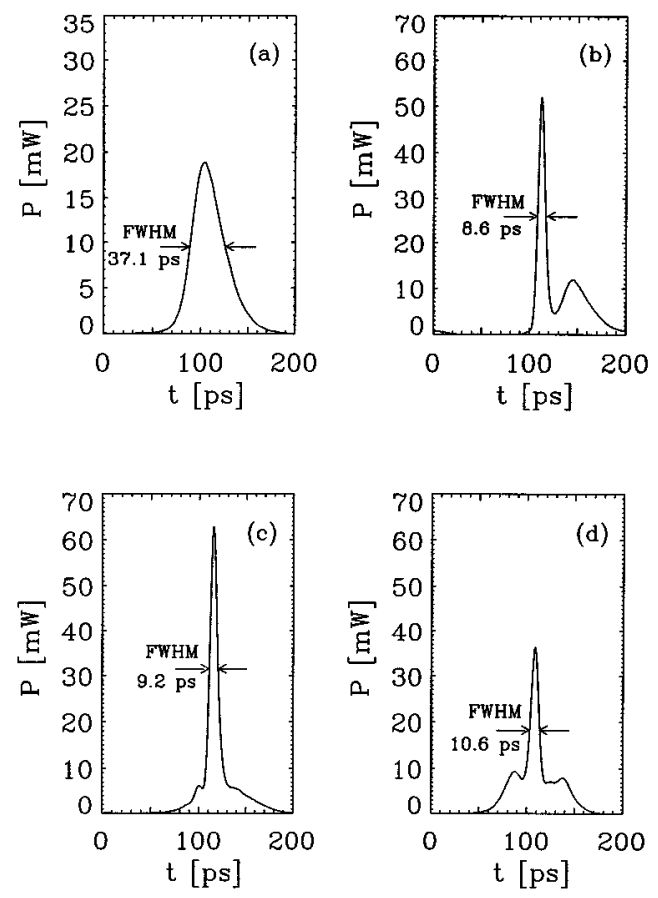

FIG. 2. Output optical pulses obtained from numerical integration of Eqs. (1)-(3) (a) from the ML, (b) from the SL for $\varphi_{s}=-100^{\circ}$, (c) the same as (b) for $\varphi_{s}=-38^{\circ}$, (c) the same as (b) for $\varphi_{s}=-10^{\circ}$

maximum and only a part of them recombine due to the interaction with the injected pulse.

Another important feature that we have observed is that when a strong pulse reduction is obtained, specially for $\varphi_{s} \sim-38^{\circ}$, the optical pulses emitted by the SL are very close to transform-limited. The product $\tau \Delta \nu \sim 0.35$, which is very close to the 0.315 of soliton pulses. In Fig. 4 it can be seen that the spectrum of the output pulse is close to the one of $\operatorname{sech}^{2}$, which we plot for comparison.

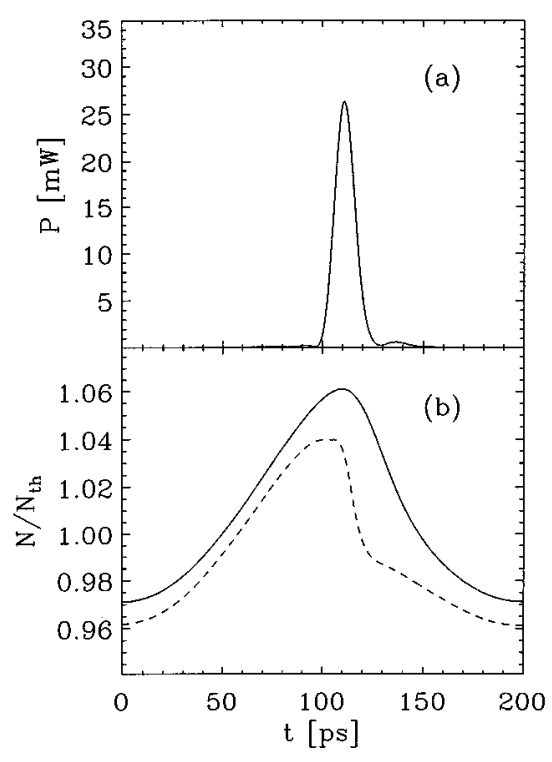

FIG. 3. (a) Injected pulse into the SL (b) carrier evolution in the SL for $\varphi_{s}=-38^{\circ}$; solid line: without pulse injection; dashed line: with pulse injection, obtained from Eqs. (1)-(3). 


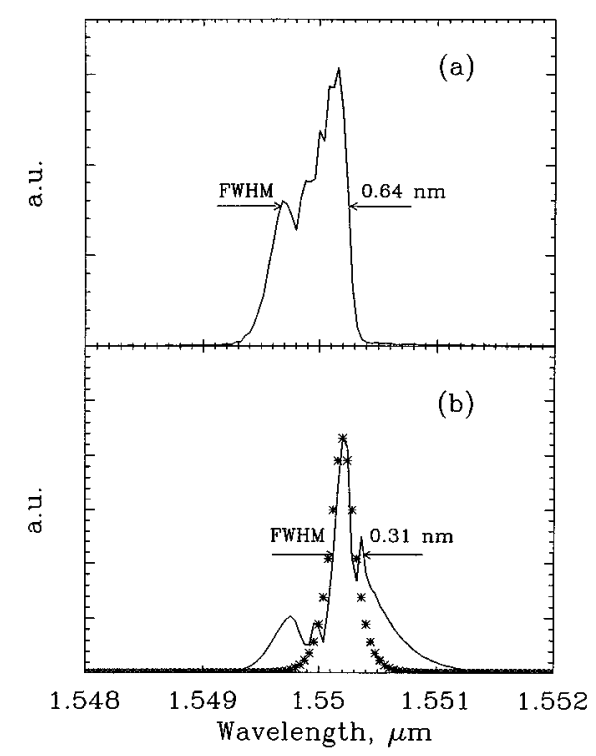

FIG. 4. Power spectrum. (a) ML or SL without pulse injection; (b) SL with pulse injection and $\varphi_{s}=-38^{\circ}$. Stars correspond to the spectrum of a $\operatorname{sech}^{2}$ pulse.

As final remark we have to mention that, as it is well known, pulses generated in both the ML and the SL are affected by spontaneous emission noise. This yields a jitter in the switch-on time of the laser ${ }^{6}$ which is also present at the moment at which the carriers reach their maximum. Consequently, the phase delay $\varphi_{s}$ which optimize pulse width reduction depends on the spontaneous emission noise. Although the range of $\varphi_{s}$, or equivalently $\Delta t_{m s}$, is large enough compared with the jitter caused by spontaneous emission, lasers with small values of $\beta$ will be better. Another way to reduce the effect of spontaneous emission noise is by using square waveform injection currents with a bias current above threshold instead of sinusoidal currents.

In conclusion, we have presented and numerically studied a simple scheme based on two single mode diode lasers coupled through a normal dispersive fiber that allows one to obtain pulses as short as 9.2 ps width, for a particular range of the temporal phase shift between the two injection currents. In addition, the obtained pulses have a maximum output power of $\sim 3$ times the output power without light injection and are very close to transform-limited as required for soliton communication systems.

This work was supported by the Comisión Interministerial de Ciencia y Tecnología, Project No. TIC93-0744. Helpful comments and discussions from E. Hernández-García, Luís Pesquera and K. A. Shore are acknowledged.

${ }^{1}$ K. A. Lau, IEEE J. Lightwave Technol. LT-7, 400 (1989).

${ }^{2}$ J.-M. Lourtioz, L. Chusseau, and L. Stelmakh, J. Phys. III France 2, 1673 (1992).

${ }^{3}$ M. Nakazawa, K. Susuki, E. Yamada, H. Kubota, Y. Kimura, and M. Takaya, Electron. Lett. 29, 729 (1993).

${ }^{4}$ C. R. Mirasso, L. Pesquera, and A. Mecozzi, IEEE Photonics Technol. Lett. PTL-5, 1455 (1993).

${ }^{5}$ M. Schell, D. Huhse, and D. Bimberg, Appl. Phys. Lett. 64, 1923 (1994).

${ }^{6}$ C. R. Mirasso, P. Colet, and M. San Miguel, IEEE J. Quantum Electron. QE-29, 23 (1993).

${ }^{7}$ H. Kawaguchi, H. Iwata, and N. Tan-No, Jpn. J. Appl. Phys. 30, L1402 (1991).

${ }^{8}$ G. Raybon, P. B. Hansen, U. Koren, B. I. Miller, M. G. Young, N. Newkirk, P. P. Iannone, C. A. Burrus, J. C. Centanni, and M. Zirngibl, Electron. Lett. 28, 2220 (1992).

${ }^{9}$ A. Weber, M. Shell, G. Fischbeck, and D. Bimberg, IEEE J. Quantum Electron. QE-28, 2220 (1992).

${ }^{10}$ K. A. Williams, D. Burns, I. H. White, W. Sibbett, and J. Fice, IEEE Photonics Technol. Lett. PTL-5, 867 (1993).

${ }^{11}$ R. Lang, IEEE J. Quantum Electron. QE-18, 976 (1982).

${ }^{12}$ G. P. Agrawal, Nonlinear Fiber Optics (Academic, San Diego, 1989). 